\section{RevistadePolítica Económica y Desarrollo Sostenible}

Centro Internacional de Política Económica para el Desarrollo Sostenible
Revista de Política Económica y Desarrollo Sostenible

EISSN: 2215-4167 • Vol. 2 (1) • Julio-Enero, 2016: 1-22

DOI: http://dx.doi.org/10.15359/peds.2-1.1

URL: http://www.revistas.una.ac.cr/politicaeconomica

Revista electrónica semestral publicada por el Centro Internacional de Política Económica para el Desarrollo Sostenible

Universidad Nacional, Lagunilla, Heredia Apartado 2393-3000

\title{
Políticas de acceso al financiamiento del micro, pequeño y mediano productor en las cadenas globales de mercancías: crédito e IED
}

\author{
Access to finance policies for micro, small and medium-sized producers in global \\ commodity chains: credit and FDI
}

\author{
Rafael Díaz Porras ${ }^{1}$ y Carlos Francisco Carranza ${ }^{2}$
}

Fecha de recepción: 24.05.2016 Fecha de aceptación: 14.11.2016 Fecha de publicación:31.12.2016

\begin{abstract}
Resumen
En este artículo se integra la teoría y conceptos del enfoque de análisis de las cadenas globales de mercancías con la teoría y conceptos de la teoría financiera del financiamiento para proponer cambios en materia de derechos de propiedad, legislación comercial, legislación financiera y de supervisión, que permitan a las instituciones financieras crear productos de financiamiento no convencionales para que pequeños productores rurales, pequeños grupos organizados de procesadores y pequeños exportadores tengan acceso al financiamiento o mejoren las condiciones en que lo reciben y así lograr que se integren de manera sostenible a las cadenas globales de mercancías. Aunque ambos enfoques conceptuales se manejan de manera independiente en la literatura, esta se analiza para determinar áreas que apoyen la propuesta de una aproximación al financiamiento desde el punto de vista de las cadenas globales de mercancías, y que la misma se convierta en un nuevo paradigma de tecnología bancaria para los agentes financieros. Se analiza la estructura y los flujos de financiamiento entre agentes de la cadena y entre agentes de la cadena y agentes financieros, nacionales e internacionales, así como el rol de la inversión extranjera directa en el financiamiento de las cadenas globales de mercancías. La propuesta para los agentes financieros de crear nuevos instrumentos de financiamiento en el marco de las cadenas globales de mercancías se construye del análisis de las características de las cadenas, de los agentes productores y de los contratos, comerciales y financieros, que garantizan la recuperación de los recursos prestados.
\end{abstract}

Palabras Clave: financiamiento, cadenas globales de mercancías, pequeños productores rurales, sistema financiero, tecnología bancaria, acceso a mercados internacionales.

$1 \quad$ Ph.D en Economía por la Universidad de Tilburg, Holanda. Catedrático del Centro Internacional de Política Económica para el Desarrollo Sostenible, Universidad Nacional (UNA) rafael.diaz.porras@una.cr

2 Máster en Economía. Ohio State University. Docente e Investigador de la Escuela de Economía, Universidad Nacional 
Revista de Política Económica • EISSN: 2215-4167

Vol. 2 (1) • Julio-Enero, 2016: 1-22

DOI: http://dx.doi.org/10.15359/peds.2-1.1

Díaz Porras y Carranza

URL: http://www.revistas.una.ac.cr/politicaeconomica

\begin{abstract}
This article connects theory and global commodity chain concepts to traditional financing theory in order to propose changes in areas such as property rights, commercial legislation, financial legislation, and supervision that would allow financial institutions to create non-conventional financial products for small scale rural entrepreneurs, organized groups of processors, and exporters to have access to finance or improve the conditions in which they receive it and thus ensure that they are integrated to the global commodity chain on a sustainable manner. Although both approaches are treated independently in literature, related publications were analyzed to determine which areas would support the proposal of having access to finance from the point of view of global commodity chains and of having this proposal become a new banking technology paradigm for financial agents. This paper analyzes the structure and financing flows between chain agents and national and international financial agents, as well as the role of foreign direct investment in the financing of global commodity chains. The proposal for financial agents to create new financing instruments in the context of global commodity chains is built as a result of the analysis of the characteristics of chains, producer agents and commercial and financial contracts, which would guarantee recovery of the resources provided.
\end{abstract}

Key Words: financing, global commodity chains, small rural farmers, financial system, banking technology, access to international markets.

\title{
Introducción
}

Los agentes productivos participantes en las cadenas globales de valor de producción de bienes agropecuarios, sin importar su tamaño o nivel de organización, necesitan fondos para financiar las operaciones corrientes de sus negocios, la inversión en renovación y el mejoramiento del equipo y la maquinaria, en innovación de productos y procesos y en expansión o penetración de nuevos mercados; en resumen, necesitan financiamiento para ser rentables y sostenibles. El financiamiento se necesita a lo largo del ciclo de vida de la empresa y puede provenir del agente mismo (autofinanciamiento) en la forma de ahorros personales (Carranza y Díaz, 2005, p. 55) y empresariales como utilidades retenidas. El capital que mantienen las empresas en forma de capital de trabajo neto (diferencia entre activo circulante y pasivo circulante) es generalmente utilizado para la operación o giro normal comercial en una base diaria, y corresponde con un uso continuo de fondos, en tanto que los fondos que mantienen en forma de utilidades retenidas se utilizan regularmente para financiar gastos mayores y discretos de inversión que deben ser amortizados a lo largo de la vida útil del activo y que pueden tener efectos importantes en productividad.

El financiamiento también puede provenir de fuentes internas a la cadena, conocido como financiamiento interno, en la forma de capital de trabajo suministrado por agentes atrás (proveedores, cuentas por pagar) o adelante (compradores, adelanto sobre cosecha) del agente. El financiamiento que proporcionan los agentes dentro de la cadena a otros agentes de la cadena, tiene como propósito conservar el flujo de mercancías para mantener las operaciones productivas, de las cuales se obtiene un beneficio o ganancia comercial. Por ello, agentes productores de la cadena están dispuestos a otorgar crédito a sus proveedores o compradores, 
Revista de Política Económica • EISSN: 2215-4167

Vol. 2 (1) • Julio-Enero, 2016: 1-22

DOI: http://dx.doi.org/10.15359/peds.2-1.1

Díaz Porras y Carranza

URL: http://www.revistas.una.ac.cr/politicaeconomica

donde las condiciones de conocimiento del "cliente" y de conocimiento de la cadena les permiten establecer "contratos" financieros y condiciones de crédito razonables (tasas de interés, costos de análisis de préstamos, control de los desembolsos, selección de garantías, cumplimiento, entre otros). Esta situación, en general, configura mecanismos de crédito más favorables y oportunos que los provenientes de fuentes de financiamiento externas a la cadena, otorgado por agentes financieros especializados en intermediación financiera, cuya actividad generadora de ingresos es la constitución de crédito en distintas formas, el seguimiento, monitoreo y control del uso de los recursos y cumplimiento de los contratos financieros. El financiamiento externo proviene de unidades económicas externas a la cadena, como bancos, financieras y cooperativas y de inversionistas privados en los mercados de valores, como personas físicas, fondos de pensiones, fondos de inversión y de fondos públicos de desarrollo.

Tanto el financiamiento interno como el externo pueden extenderse a través de las fronteras en ambas direcciones (el país donde se origina la cadena y el país donde tiene lugar el consumo final), catalogándose como financiamiento nacional o internacional, según el domicilio fiscal del ente otorgante, no del ente que recibe el crédito. Asimismo, ningún agente productivo se desempeña en un vacío social, es parte de un grupo de agentes, que pueden competir o cooperar, y de una cadena de mercancías.

Las cadenas de valor "se definen como redes interrelacionadas de productores y comercializadores que están relacionados en una secuencia de creación de valor y operan bajo la orientación que integra la producción primaria, la transformación, la comercialización y el uso final” (Gereffi y Korseniewicz, 1994, p.1-14). Según Hess et al., (2007, p. 5), la cadena de valor es también una "secuencia de actividades de negocio (funciones) que van desde el suministro de los insumos específicos para la producción de un producto particular, a través de la transformación y el mercadeo, hasta la venta final del producto al consumidor (...)" es un "grupo de empresas (operadores) que realiza esas funciones, i.e. productores, procesadores, comercializadores y distribuidores de un producto particular. Las empresas están ligadas por medio de una serie de transacciones de negocio a través de los cuales el producto pasa de la etapa de elaboración primaria hasta el consumidor final (...)" y también podría ser un "modelo de negocios para un producto comercial particular. Este modelo de negocios permite alcanzar a determinado grupo de clientes con el uso de una tecnología dada y una forma particular de coordinar la producción y el mercadeo entre varias empresas (...)”3. Por su parte, 迆en $(2007$, p. 1), señala que una cadena de valor es una "progresión de actividades que agregan valor, empezando con la materia prima y terminando con la venta del producto terminado o servicio".

De esa forma, la cadena de valor no resulta en una entidad legal sujeta al análisis de valor crediticio por agente financiero alguno, ni tiene personería jurídica para la firma de contratos financieros o contratos de financiamiento; sin embargo, el conocimiento y comprensión de la misma sí resulta relevante para la aceptación o rechazo de solicitudes de financiamiento que, en

3 Traducción de los autores. 
diversas formas, son planteadas por distintos tipos de agentes productivos a lo largo de la cadena, desde el proveedor de los insumos iniciales hasta el consumidor del producto final, e incluso por quienes tienen como actividad productiva corriente el tratamiento de los desechos del consumo. La dirección del financiamiento puede cruzar las fronteras en ambas direcciones.

Ahora bien, la pregunta relevante es ¿cómo mejorar el acceso y las condiciones crediticias de estos agentes productivos? Para ello, se debe explorar los mecanismos de aseguramiento y la creación de mercados de contratos a futuro donde la información sea más confiable y se conozcan los costos de enfrentar el riesgo por parte de estos agentes.

La literatura reseña la relación entre acceso al financiamiento externo y mayor capacidad de crecimiento, mejoramiento de la competitividad y el upgrading. La mayor preocupación en esta se relaciona con el acceso al crédito por parte de los productores de manera individual, resultado de la evaluación del vector de características socioeconómicas y financieras del mismo, pero sin considerar el contexto de las cadenas. La literatura muestra las condiciones y factores del productor que le garantizan una probabilidad máxima de obtener y mantener acceso al financiamiento externo y con las mejores condiciones; el conocimiento profundo de ese conjunto de condiciones señala el grado de experiencia de los agentes financieros en el proceso de análisis, selección, seguimiento y monitoreo de sus préstamos.

Akin y Fries (2004) señalan que el reto de las finanzas rurales consiste en complementar el trabajo de las instituciones financieras, elaborando productos que ofrecen a las empresas rurales, con elementos de la cadena de valor en que participan y con elementos de las oportunidades y restricciones que dichos productores enfrentan. No señalan, sin embargo, propuestas concretas para alcanzar tal cometido. Meyer (2007) sugiere que el análisis de cadenas de valor, en general, y el análisis de cadenas agrícolas de valor, en concreto, puede ayudar a identificar intervenciones que expandan los servicios financieros a productores y comunidades rurales, pero no propone mecanismos o procedimientos de aplicación por los agentes financieros para incorporar elementos de las cadenas en la selección y el análisis del crédito, o en el diseño y ejecución de contratos.

En contraste, los procedimientos de rutina de la tecnología bancaria tradicional se basan en determinar el nivel o clase de riesgo que el prestatario agropecuario representa (UNCTAD, 2004). Esta misma fuente indica que, en el tanto los productores solicitantes de crédito mantengan el mismo nivel de desempeño en las cadenas agrícolas de suministros, los bancos verán reembolsadas sus operaciones de préstamo. No señala, sin embargo, cómo calificar, bien o mal, dicho desempeño en las cadenas; otro elemento ausente en la tecnología bancaria, y se señala que "el riesgo de que los prestatarios cesen de desempeñarse apropiadamente es mitigado por el uso por parte de los bancos de un rango de relativamente nuevas herramientas de manejo de riesgo" (ibíd).

Johnston (2007) reconoce un importante elemento de la actividad de financiamiento que surge de la dinámica de las cadenas de valor: los agentes de la cadena que prestan servicios de financiamiento a otros agentes deben resolver el mismo problema que los agentes financieros; 
esto es, determinar la capacidad y voluntad de pago de sus clientes prestatarios, tema que se desarrolla en detalle en este artículo. En esta misma línea, Sucre Reyes (2014) cita la necesidad de fortalecer el financiamiento indirecto en las cadenas de valor por medio de los agentes financieros, como una medida para enfrentar limitaciones en el financiamiento de estas.

En el tema de financiamiento, existe otro conjunto distinto de condiciones y factores que, sumados a la condición de tener acceso al financiamiento, pueden conducir a un proceso de concentración del control en cada uno de los distintos mercados de una cadena global de mercancías. Este otro conjunto está compuesto por elementos propios del agente productor y por elementos propios de la cadena, probablemente de carácter fortuito, coyuntural u ocasional, y donde lo más relevante es la reacción de uno de los agentes de la cadena ante ellos.

Finalmente, el análisis en este artículo considera las condiciones de financiamiento que contribuyen a la sostenibilidad de las cadenas y propuestas de operación bancaria, así como de regulación financiera y comercial de interés para agentes productores, agentes financieros y autoridades reguladoras. A pesar de la heterogeneidad y complejidad de las cadenas globales de mercancías, se propone una clasificación para que sea usada como instrumental de análisis de crédito.

\section{Cadenas globales de mercancías y financiamiento}

\subsection{Cadenas globales de mercancías y financiamiento vía crédito}

El acceso de los agentes productores al financiamiento externo es una condición necesaria, aunque no suficiente, para su crecimiento y sostenibilidad, así como para el crecimiento y sostenibilidad de las cadenas globales de mercancías. Sin embargo, bajo los procesos y métodos bancarios actuales, en muchos países en desarrollo agentes productores y segmentos completos de las cadenas no logran obtener acceso al financiamiento externo. Así pues, efectuar los cambios legales, introducir innovaciones en la tecnología bancaria, en los instrumentos, contratos, tipos de garantía, seguros, entre otros, y crear las condiciones para que el financiamiento externo esté disponible para los agentes productores es una tarea conjunta de agentes productores, agentes financieros, autoridades de gobierno y agencias reguladoras. Ambos sistemas, el financiero y la cadena global de mercancías, se encuentran para determinar el acceso al financiamiento externo.

La cadena global de mercancías es una categoría conceptual de análisis, no es una entidad legalmente constituida que pueda ser objeto de préstamos, subsidios u otro contrato comercial; los agentes que la componen sí. El fracaso de un agente individual dentro de un segmento de una cadena no genera el fracaso de la cadena en su totalidad, pero el fracaso, o tan solo el mal funcionamiento de un segmento completo, sí puede generar el fracaso de toda la cadena en alguna de sus dimensiones, económica, social y ambiental, o en todas simultáneamente. 
Para los agentes productivos en la cadena, el financiamiento, tanto interno como externo, responde a las necesidades de corto plazo de capital de trabajo e inventarios para hacer frente a la compra de semillas e insumos, la siembra y mantenimiento del cultivo, la cosecha y el procesamiento primario y la recolección de información de acceso a mercados. En el largo plazo, el financiamiento se requiere para inversión de capital, innovación y promoción de la competitividad (Carranza y Díaz, 2005, p. 53). Con ello se logra generar ganancias, mantener la posición en el mercado, penetrar nuevos mercados y crear mecanismos de subordinación con agentes anteriores y posteriores en la cadena para aumentar el grado de control de precios y rentas en esta.

Las cadenas globales de mercancías representan un medio para la generación de ingresos de los agentes agropecuarios, algunos de los cuales son pequeños productores rurales de tipo familiar e informal, en términos económicos, legales, administrativos y contables, en sus segmentos iniciales. Estos segmentos de productores agropecuarios individuales, de cooperativas $\mathrm{o}$ asociaciones de productores consolidando oferta de producto y de procesadores primarios, son del interés de las autoridades, ya que resulta de gran utilidad económica y social que dichos agentes logren integrarse exitosa y sosteniblemente a una cadena global de mercancías. La disponibilidad de financiamiento oportuno y a un costo adecuado es uno de los factores que contribuyen a la sostenibilidad de las cadenas, definida solamente como la permanencia de la misma, sin considerar otras características.

En KIT et al. (2010, p. 243-245) se establecen tres escenarios idóneos para el financiamiento de las cadenas: para formar nuevas cadenas, para mejorar la liquidez en cadenas existentes y para fomentar la inversión. Contar con indicadores apropiados para clasificar las cadenas es relevante para los agentes financieros como primer paso en el proceso de análisis, selección, aprobación y rechazo de solicitudes de financiamiento. Tanto la imagen pública y el desempeño social y ambiental como el desempeño y las variables económicas, financieras, contables, administrativas, de producción y mercadeo, deben ser valorados por los agentes financieros, así como la disponibilidad de activos de garantía y el historial crediticio.

El desempeño de las cadenas globales de mercancías se caracteriza con distintos indicadores. El desempeño ambiental se caracteriza con indicadores sobre emisiones y eficiencia energética; mientras que el desempeño económico y social se caracteriza con indicadores como la edad o permanencia de la cadena, su extensión geográfica nacional e internacional, tamaño, estabilidad y tendencias del mercado de consumidores finales, la existencia de instituciones públicas y privadas normativas, el número de agentes productores y su grado de homogeneidad, la calidad empresarial, experiencia e historial de crédito de los productores, el nivel de equidad en la distribución de rentas generadas a lo largo de la cadena, el valor agregado y la generación de rentas, la distribución de riesgos entre agentes y segmentos, la tipología de procesos productivos y el grado de transformación y diferenciación de productos, entre otros.

En las cadenas maduras y desarrolladas las solicitudes de financiamiento no requieren por parte del agente financiero una calificación y clasificación previa de la cadena. Sin embargo, para 
las solicitudes de financiamiento que provienen de agentes en cadenas creadas para producir y comercializar productos nuevos, el agente financiero debe recopilar información sobre los indicadores arriba mencionados para analizar y clasificar la cadena previo al análisis individual del cliente. Según la clasificación otorgada a la cadena, ya sea alta, media o baja, el potencial para el otorgamiento de recursos de financiamiento será también alto, medio o bajo. ${ }^{4}$

Los agentes productores en cadenas maduras tienen un potencial de recibir financiamiento mayor que los agentes en las cadenas de menor desarrollo. Chalmers et al. $(2007$, p.36) señalan la variable $f(Z)$ como el grado de madurez y eficiencia de la cadena a la que está integrado el productor como una de las variables que determinan el acceso al financiamiento externo. Según estos mismos autores, el acceso al crédito se explica mediante la ecuación: acceso al financiamiento $=F[P, f(Z), C, E]$, donde $\mathrm{P}$ es un vector de características personales del productor que incluyen tamaño y ubicación de la finca, educación del propietario, distancia al mercado y grado de tecnificación; $\mathrm{f}(\mathrm{Z})$ es el grado de madurez y eficiencia de la cadena a la que está integrado el productor; $\mathrm{C}$ es el grado de competencia, eficiencia y estructura del mercado financiero; y por último, E representa las condiciones macroeconómicas.

En ausencia de acceso al financiamiento, los agentes productores se ven obligados a recurrir al autofinanciamiento o acumulación de ahorros propios o retención de utilidades. En segundo lugar, recurren, si tienen acceso, al financiamiento interno. Este financiamiento, suministrado por otros agentes productores a quienes, como lo señala Johnston (2007), los motiva el fin de asegurar los ingresos de una operación productiva y no el fin de obtener ingresos financieros como actividad normal o principal de negocio. ${ }^{5}$ Tanto Zeller (2005) como Akin y Fries (2004) establecen que estos agentes, aunque no se dedican de manera expresa a la actividad crediticia, por el papel que juegan dentro de la cadena, tienen un mayor grado de conocimiento de los agentes que reciben sus recursos crediticios. En tercer lugar, recurren al financiamiento que proporcionan agentes financieros nacionales y extranjeros, externos a las cadenas globales de valor, que se dedican al suministro de dinero y capitales como su giro normal de negocio, en el marco de la legislación bancaria y comercial del país.

Una cuarta forma de financiamiento puede tener lugar en los mercados primarios de bonos y en los mercados primarios de acciones, en el marco de la legislación de valores del país, donde inversionistas privados compran papeles de esas empresas o agentes productores. Los bonos por un plazo y costo definidos; las acciones por un plazo indefinido, además sujetas a un rendimiento variable.

Los agentes financieros externos a la cadena están mejor preparados en cuanto a disponibilidad de fondos prestables y conocimientos de tecnología bancaria, pero menos

4 La institución financiera debe construir sus propios criterios para dar a cada indicador la categoría de alto, medio y bajo, para construir las ponderaciones (homogéneas o diferenciadas) apropiadas y para asignar a cada tipología de cadena un potencial de crédito distinto sobre las otras.

5 Los agentes de la cadena que proporcionan financiamiento a sus compradores y/o vendedores (financiamiento interno) no tienen como objetivo de esa actividad la generación de rentas financieras, sino el aseguramiento de compras o ventas del producto o productos principales de su actividad productiva empresarial (Johnston, 2007) 
preparados en términos de conocimiento de la cadena y de los agentes de la misma. El análisis de crédito que tienen que efectuar los agentes financieros para aceptar o rechazar clientes, así como la selección y diseño de instrumentos de financiamiento y de cláusulas de contrato de préstamo, deben tomar en cuenta no solo las características del agente productor que solicita financiamiento, sino también las características de la cadena global de la mercancía en que opera el solicitante: un segmento anterior o posterior que signifique un cuello de botella en el flujo del producto puede constituir una amenaza para la recuperación de los recursos dados en préstamo.

Considerar el modelo de cadenas globales de mercancías en el análisis tradicional de crédito abre un espacio enorme para el diseño de recomendaciones técnicas para los agentes financieros, los reguladores y los creadores de leyes y políticas, en términos del diseño de herramientas de análisis crediticio y de metodologías de monitoreo y control post-contrato del crédito. El marco legal y las regulaciones financieras y bancarias deben permitir que los contratos respondan a la dinámica de la cadena.

En el proceso de selección y rechazo de solicitudes de financiamiento el agente financiero requiere información empresarial de sus solicitantes o agentes productivos para clasificarlos en categorías según su calidad y capacidad empresarial (CCE). Dicha información es indispensable para determinar la capacidad y disponibilidad de pago de los solicitantes. La calidad y capacidad empresarial depende del grado de información, conocimiento y experiencia empresarial que tiene el agente de la actividad que desempeña. A mayor nivel del índice de CCE, más probabilidad de éxito del agente productor; asimismo, conocer bien la CCE del solicitante resulta crítico para el éxito del agente financiero.

Para los distintos efectos de este artículo, los agentes financieros clasifican a los agentes productores de acuerdo con el valor del índice CCE en tres categorías: nuevo, medio y establecido. Los agentes productores establecidos tienen la mayor probabilidad de tener acceso al financiamiento externo y en mejores condiciones que los demás.

\subsection{Cadenas globales de mercancías y financiamiento vía IED}

El financiamiento de carácter interno (entre agentes de la cadena) puede extenderse a través de las fronteras, tanto de agentes extranjeros a nacionales como de agentes nacionales a extranjeros. Cuando la magnitud de ese financiamiento alcanza niveles mayores al $10 \%$ del capital social de la empresa receptora es de carácter no devolutivo y tiene como objetivo hacerse del control en la toma de decisiones; así, el financiamiento interno se convierte en inversión extranjera directa, (IED).

Los recursos financieros que utilizan los agentes de la cadena radicados en el extranjero provienen de sus utilidades retenidas, del financiamiento de agentes financieros radicados en su mismo país, del financiamiento de agentes financieros extranjeros y de los mercados internacionales de capital. Para identificar claramente a los agentes financieros es conveniente utilizar la distinción de domicilio fiscal en lugar de la ubicación geográfica. 
Para efectos de análisis de cadenas globales de mercancías, la denominación de nacional para un segmento o agente productor o para un sistema o agente financiero, se refiere a la localización geográfica de las fases de siembra, procesamiento primario y exportación. La denominación de internacional, extranjero o foráneo se refiere a los segmentos y agentes fuera de las fronteras nacionales hasta alcanzar los mercados de consumo final. Este financiamiento podría considerarse como crédito de carácter externo porque la fuente primaria de los recursos son agentes financieros y no agentes productores, pero dado que el crédito se otorga por agentes de la cadena a otros agentes de la cadena y que no tiene como propósito la generación de ingresos financieros sino el aseguramiento de giro normal de los negocios, sigue siendo financiamiento interno, aunque el origen de los recursos sea financiamiento externo.
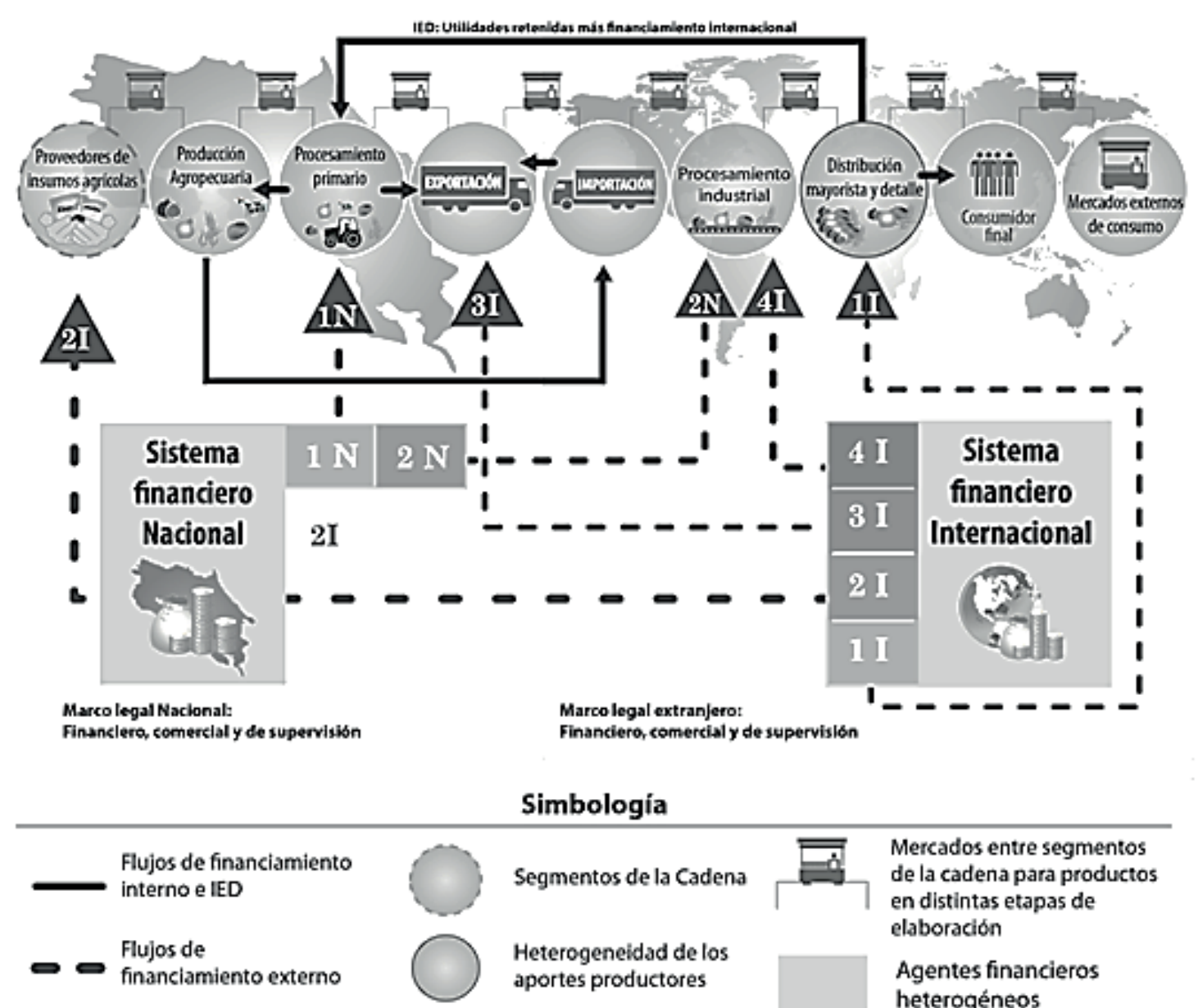

\section{Simbología}

Segmentos de la Cadena

Heterogeneidad de los aportes productores

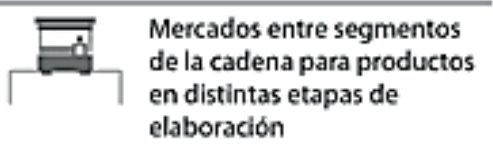

Agentes financieros heterogéneos

Figura 1. Flujos de financiamiento e IED en las cadenas

Nota: En simbología léase correctamente "heterogeneidad de los agentes productores"

Fuente: Carranza y Díaz, 2015. 
La Figura 1 representa la cadena global para un producto cuya fase de siembra y cosecha está a cargo de micro, pequeños y medianos productores rurales; en el mismo medio rural podría encontrarse también alguna forma de procesamiento primario. La línea media señala la frontera que indica no solamente el punto de exportación a otros países donde se efectúan otros tipos de procesamiento, re-empaque, re-exportación y el consumo final, sino también la frontera fiscal o tributaria que determina la nacionalidad de los agentes, tanto financieros como productores. Los segmentos nacionales e internacionales, representados con pequeños círculos, están compuestos por agentes productivos con distintas características económicas y distintos niveles de escala ${ }^{6}$. Los flujos de financiamiento interno, entre agentes de la cadena, se muestran con flechas horizontales, en ambas direcciones y entre segmentos adyacentes.

Los agentes financieros internacionales trasladan fondos desde los mercados de dinero y de capitales a empresas radicadas en sus propios países y a empresas radicadas en el extranjero. El financiamiento internacional llega a empresas radicadas en el extranjero por tres diferentes rutas. La primera, los agentes financieros internacionales dan financiamiento a empresas de sus mismos países quienes complementan sus utilidades retenidas e invierten en el extranjero para la adquisición de activos fijos o participaciones accionarias mayores al 10\% del capital social de empresas en el país donde se encuentran los segmentos iniciales de la cadena. Este corresponde a un flujo de inversión extranjera directa (IED) en las cadenas globales de mercancías (ruta 1I). La segunda, como financiamiento a agentes financieros nacionales quienes otorgan préstamos a agentes productores nacionales (ruta 2I). La tercera, corresponde al flujo 3I, que va desde los agentes financieros internacionales hacia agentes productores nacionales grandes y medianos (ruta 3I). Cualquiera de estos recursos de financiamiento proveniente de un agente financiero internacional puede terminar como financiamiento interno entre agentes de la cadena. Los préstamos de agentes financieros internacionales a empresas de su mismo domicilio fiscal ocurren de manera directa (ruta 4I). Este se distingue de la ruta 1I en que no termina como IED.

Aunque con menos frecuencia, porque los recursos de capital nacionales son limitados y el conocimiento, la tradición y la experiencia en el manejo de operaciones financieras internacionales son relativamente inapropiados, los agentes financieros nacionales pueden efectuar operaciones de financiamiento a empresas internacionales en las tres formas descritas arriba (flujo $2 \mathrm{~N}$ ). Por lo regular, los agentes financieros nacionales dan financiamiento a agentes productores nacionales (flujo $1 \mathrm{~N}$ ).

La inversión y el upgrading de agentes productores en las cadenas globales de mercancías se puede lograr a través de la inversión extranjera directa o a través del financiamiento vía crédito otorgado por agentes financieros externos, tanto nacionales como internacionales. Los determinantes del crédito en relación con las características de los agentes productores, sus mercados y la cadena, son el objeto de estudio de la sección siguiente, con el propósito de extraer propuestas de política pública, propuestas de gestión bancaria y propuestas de gestión empresarial.

6 Las características económicas y de escala de los agentes productores en un mismo segmento de una cadena global pueden diferir de un país a otro. 
El acceso al financiamiento externo con oportunidad y suficiencia se da ya sea mediante instrumentos convencionales, como crédito, acciones y bonos, ${ }^{7}$ o mediante instrumentos no convencionales como el leasing, el factoring, el capital de riesgo, las franquicias y la titularización, cada uno de ellos regulado por normativas legales y comerciales específicas ${ }^{8}$. Los instrumentos de financiamiento interno, como el crédito comercial (trader credit), los adelantos sobre cosechas (contract farming and outgrowers schemes) y el crédito contra recibos de depósito (warehouse receipts $)^{9}$, le permiten al agente productor no solo mantener las operaciones corrientes, sino también invertir en capital e innovar, lo cual favorece las posibilidades de mejorar la posición competitiva dentro de la cadena, mejorar la participación en las rentas generadas en la cadena y eventualmente aumentar el poder o adueñarse del control del segmento de la cadena en el cual opera.

Las condiciones del crédito se refieren a montos, plazos, planes de desembolso, tasa de interés, comisiones, gastos legales, avalúos, garantías, monitoreo, seguimiento y control del uso de los recursos y algunos otros costos implícitos o costos de transacción ${ }^{10}$, y las cláusulas del contrato que, de acuerdo con la legislación y marco legal, se diseñen para crear los incentivos y las amenazas para lograr el cumplimiento de los pagos del principal e intereses. Cuanto más favorables sean las condiciones del financiamiento externo o crédito, mayor es la probabilidad de que el crédito tenga un efecto positivo sobre el control.

El financiamiento interno se realiza en las siguientes formas que se detallan a continuación. El primer tipo se origina en las cuentas por cobrar y cuentas por pagar de unos y otros agentes, por compras y ventas a crédito, usualmente a plazos de 30,60 y 90 días sin cobro de tasa de interés y donde la garantía está constituida por las facturas. El crédito comercial ligado al suministro de insumos (trader credit) son los adelantos de efectivo hacia atrás que le permiten al productor hacer frente a gastos de cosecha. Los contratos de compra de cosecha condicionan el suministro de insumos a un contrato de venta de la cosecha (outgrowers scheme and contract farming), tomando la forma de un pago adelantado por la cosecha y también cuando el comprador recibe la cosecha pero necesita un plazo para procesarla y exportarla, o solo para exportarla, y no puede efectuar el plazo de contado, en cuyo caso recibe un crédito del vendedor. En los adelantos de efectivo por cosecha tampoco hay cobro de tasa de interés, pero se fija el precio del producto de la cosecha y la garantía es la cosecha misma. El riesgo de una cosecha inferior al monto del financiamiento debe ser negociado entre ambas partes. Los recibos de almacenamiento (warehouse receipts) en recibidores independientes certificados sirven para garantizar el crédito de otros agentes productores. Estos recibos de almacenaje de productos no perecederos, emitidos por centros de acopio confiable y seguro, le permiten a las instituciones financieras usar el inventario almacenado como garantía de préstamos.

7 Aunque con menor importancia se utilizan también los bonos convertibles y warrants.

8 Las características de estos instrumentos no son objeto de este capítulo.

9 Akin y Fries (2004), traducción de los autores.

10 Se refiere a gastos incurridos por el solicitante, como gastos de traslado o el tiempo laboral y empresarial usado en viajes y visitas al banco. Dependen de la estructura tecnológica y eficiencia del banco y de la cercanía entre el banco y el solicitante. 
El crédito comercial (Akin y Fries, 2004) consiste en el otorgamiento de préstamos estacionales de corto plazo entre compradores y vendedores de insumos o de productos y está típicamente relacionado con cadenas de valor de commodities. La relación entre compradores y vendedores tiene mayor grado de temporalidad y orientación por los precios que en el caso del contract farming $y$ outgrower schemes. En estos últimos, los vendedores se encuentran en una relación más formal o cautiva con el comprador, quien a cambio se compromete usualmente a proporcionar servicios adicionales como la asistencia técnica. Este nivel mayor de compromiso resulta más apropiado entre compradores y vendedores de productos de alto valor o para especialidades.

Cuando el financiamiento es otorgado por un agente productor a otro, la cercanía en términos económicos con quien recibe el crédito, origina un mayor grado de conocimiento sobre la actividad económica e historial del solicitante y mejora la efectividad de imponer castigos comerciales si hubiera incumplimientos de contrato (prenda sobre herramientas y existencias de cosecha, rompimiento de la relación empresarial con el proveedor y cesación del crédito para futuras operaciones), y hacen que el crédito entre agentes productores sea más eficiente que el crédito otorgado por agentes financieros externos de la cadena. Estos últimos, por tamaño y falta de especialización, tienen índices de información menores en todos los aspectos comerciales enumerados. El reto de los agentes financieros externos y sistema financiero formal es cómo superar esos inconvenientes y poner un volumen mayor de recursos al alcance de los agentes productores en las cadenas e incrementar el grado de acceso al financiamiento de un mayor número de agentes.

El análisis de las solicitudes de financiamiento que realizan los agentes financieros se da bajo condiciones de información incompleta, imperfecta y asimétricamente distribuida entre él y el agente productor. Esa tarea técnica es indispensable para hacer la mejor estimación posible de la capacidad de pago (aspecto técnico-empresarial) y de la disponibilidad de pago (aspecto histórico-moral) de cada solicitante, en el marco de su presupuesto operativo, recurso humano especializado y tecnología bancaria, para determinar la probabilidad de incumplimiento de pago o mora (default) de cada solicitud de financiamiento. Para efectuar una labor financiera efectiva y eficiente, el agente financiero debe tener un conocimiento especializado de la cadena global donde tiene negocios un agente productor que le solicita financiamiento y elaborar un índice de valor crediticio de cada cliente con base en el vector de características del mismo ${ }^{11}$.

Para reducir las pérdidas por mora a niveles aceptables en la industria bancaria o establecidos por las instituciones de supervisión financiera prudencial es necesario que los agentes financieros tengan amplio conocimiento de la actividad bancaria y de la tecnología de préstamos, de las cadenas globales de mercancías en que operan sus clientes y de las características individuales de cada uno de ellos en los distintos segmentos. Shwedel $(2007$, p.24) llama "financiamiento en cascada" al financiamiento bancario integral de agentes productores o empresarios en segmentos distintos de una cadena. Refiriéndose a la cadena global de las flores en la cual participan empresarios mexicanos ese autor dice:

11 Por esa razón las instituciones financieras deben contar con equipos humanos especializados en distintas actividades económicas o escoger áreas de especialización. 
“(...) la comercialización de flores se ha convertido en un negocio especializado y global. Para el agricultor representa un producto de alto valor y requiere de inversiones cuantiosas e importantes. Una hectárea de invernadero nos cuesta en México más o menos un millón de dólares, entonces es un negocio altamente especializado. ¿Estamos dispuestos a financiarlo o no? En este caso la repuesta ha sido afirmativa, porque estamos presentes en "cascada", lo que permite un conocimiento profundo del negocio. Estamos financiándole al agricultor sus necesidades de capital de trabajo; financiando equipo, tecnología y también estamos financiando a los vendedores de equipo. Estamos financiando al agricultor porque lo conocemos a él y al sistema de comercialización. El agricultor en lo fundamental manda su producto a un mercado de subasta en Holanda y somos los que financiamos el mercado de subastas; también financiamos mucho a los compradores. Tenemos amarrado el financiamiento de la cadena (...)"

Además, todo lo anterior es necesario para diseñar productos financieros de crédito y ofrecerlos a los distintos demandantes. En cada tipo de instrumento debe mediar un contrato que establezca incentivos legales y monetarios para prevenir el incumplimiento o default, y para, finalmente, señalar garantías y procesos legales de recuperación en caso de falta de pago, para lo cual la legislación comercial y bancaria normando todo lo relativo a los derechos de propiedad y seguridad jurídica es crucial; igualmente importante resulta la estructura de supervisión prudencial.

\section{Financiamiento en el marco de las cadenas globales: una estructura analítica para el diseño de tecnologías bancarias}

En esta sección las cadenas globales de mercancías se denotan A, B, C... Z y los agentes productivos en cada una de ellas como aj, bj... zj; donde a, b, c... z representan los segmentos que componen cada cadena y $\mathrm{j}$, para $\mathrm{j}=1,2 \ldots, \mathrm{n}$ representa a un agente productor cualquiera en cualquier segmento, de modo que $\mathrm{B}(\mathrm{c} 3)$ representa el agente productor 3 del segmento $\mathrm{c}$ de la cadena B. Por otro lado, los agentes financieros externos se denotan por Fk, $\mathrm{k}=1,2 \ldots, \mathrm{m}$, donde $m$ representa el número de agentes financieros en el mercado, tanto nacionales como extranjeros y de diferentes grados de formalidad legal. Para simplificar el análisis se propone clasificar a los agentes en las tres clases antes indicadas: nuevo, medio y establecido; y las cadenas también en las mismas categorías: nueva, media y establecida.

La información (I), el conocimiento (C) que el agente productor tenga de su producto, proveedores y compradores, producto final y compradores finales y de toda la cadena, y la experiencia empresarial (E) son valorados en la determinación del índice de valor crediticio ${ }^{12}$ y también en la explicación del grado de control que puede adquirir sobre el segmento de la cadena o sobre la cadena. Estas tres variables I, C y E son del interés del productor j, de sus socios

12 Esta información se complementa con el vector X de características del solicitante de crédito y comprende variables como tamaño económico, riqueza, edad, educación y tamaño de la familia en el caso de empresas rurales familiares (Carranza 2001; 2002). 
comerciales $(j-1, j+1)$ en los segmentos anterior y posterior y de los agentes financieros $(F)$ para decidir su acceso o rechazo a los recursos financieros.

Algunos agentes de la cadena conocen bastante bien las variables I, C y E de otros agentes para brindarles financiamiento interno a través de contratos comerciales (CC). En el contrato comercial se establecen la cantidad y precio de la mercancía así como el plazo del financiamiento (V), pero no existen otros costos o condiciones como intereses o garantías. Un contrato comercial entre el agente $\mathrm{j}$ y el agente $\mathrm{j}+/-1$ se denota con la expresión: $C \mathrm{Cj}, \mathrm{j}+/-1=\mathrm{f}(\mathrm{V}, \mathrm{Qj}, \mathrm{Pj})$. Este contrato no representa carga financiera por intereses para el beneficiario, ni establece garantías y mecanismos legales de cobro en caso de incumplimiento, porque el financiamiento interno contiene otros incentivos para el cumplimiento de contrato, no la amenaza de apropiación de garantías, sino la amenaza del rompimiento de la relación comercial, de la continuidad de la actividad productiva, de la cual ambos se benefician como agentes de la cadena, pero, por su posición en la cadena, se perjudica más quien recibe el financiamiento si incumple el contrato. El monitoreo y control que ejerce el agente que da el financiamiento es parte de su actividad productiva y no asigna recursos adicionales a esa tarea, los costos operativos del financiamiento a otros agentes de la cadena son parte de los costos totales de operación, no representan un costo extraordinario.

Surge una contradicción de objetivos en el productor que requiere acceso al financiamiento externo: por un lado debe mostrar transparentemente toda la información requerida por agentes financieros y tiene incentivos para revelarla, pero por otro, especialmente información que tiene carácter de secreto comercial o información que podría indicar su inexperiencia o conocimiento no quiere revelarla necesariamente. Para los agentes financieros, a mayor transparencia de la información, mayor probabilidad de otorgamiento de crédito a agentes productores buenos sujetos de crédito; asimismo, para los agentes productores, a menor transparencia de secretos comerciales e información valiosa en el éxito del negocio, mayor probabilidad de éxito comercial, y con algún grado de probabilidad, dicha información no es trasladada de manera completa en las solicitudes de crédito formal. Mucha de la información comercial que tiene el agente productor es cualitativa y basada en información incompleta de sus socios y competidores. Algunos solicitantes podrían deliberadamente dar al agente financiero información que sobreestime el tamaño de las ventas y su participación de mercado, subestimar gastos operativos y ocultar riesgos comerciales que solamente ellos conocen.

Esta contradicción plantea un reto para las autoridades de regulación financiera y comercial así como para los agentes financieros en el diseño de políticas e instrumentos financieros para hacer la información más transparente y completa. Es necesario revisar los derechos de propiedad, la legislación mercantil y las normas de supervisión financiera para encontrar mecanismos legales que permitan a los agentes financieros diseñar productos financieros innovadores ${ }^{13} \mathrm{y}$

13 La Ley 9274, Sistema de Banca para el Desarrollo en Costa Rica, es un ejemplo de cambios del marco legal para permitir la creación de otros instrumentos y la aceptación de clientes que no califican bajo la legislación financiera y marco regulatorio vigentes. Sin embargo, los nuevos instrumentos deben alcanzar no solo a los agentes cubiertos en dicha ley, sino a todos los que presenten problemas de riesgos y garantías según la supervisión actual. 
Revista de Política Económica • EISSN: 2215-4167

Vol. 2 (1) • Julio-Enero, 2016: 1-22

DOI: http://dx.doi.org/10.15359/peds.2-1.1

Díaz Porras y Carranza

URL: http://www.revistas.una.ac.cr/politicaeconomica

crear compromisos contractuales entre distintas cédulas jurídicas en diversos segmentos de la cadena para el desempeño comercial y que puedan generar incentivos cruzados y solidarios de cumplimiento de los pagos de reembolso del financiamiento. Por ejemplo, que el financiamiento a agentes dentro de un segmento no se vea obstaculizado por el fracaso de agentes productores en segmentos posteriores de la cadena por ausencia de acceso al financiamiento.

Con información imperfecta e incompleta acerca de las variables $\mathrm{Ij}$, Cj y Ej del agente productor, información de las características del productor $(\mathrm{Xj})$, de las garantías reales de bienes muebles o inmuebles disponibles del productor $(\mathrm{GjD})$, del historial crediticio del solicitante $(\mathrm{Hj})$ en los registros de agencias especializadas y, especialmente, con información que caracterice la cadena global mediante el Índice de la Cadena Global (ICGj), ${ }^{14}$ el agente financiero externo asigna a cada productor solicitante un número o índice como sujeto de crédito (Wkj), determina la probabilidad de otorgarle financiamiento o rechazarlo, y las condiciones del crédito, si lo otorga.

Es probable que distintos agentes financieros (F) asignen distintos índices Wkj y probabilidades de acceso a un mismo agente productor o empresario, dependiendo de la valoración (g) que hagan de la información, de sus políticas y su tecnología bancaria. A partir esa valoración diseñarían distintos contratos financieros (CFKJ) y condiciones del financiamiento, en cuanto a monto (M), plazo (V), costo o tasa de interés (R), plan de desembolsos (D) y las garantías elegidas por el agente financiero (GE) que el agente financiero k le garantiza al productor $\mathrm{j}$. El contrato financiero (CFKJ) es una función h que determina $\mathrm{M}, \mathrm{V}, \mathrm{R}, \mathrm{D}$ y GE como resultado de la función de valoración crediticia $g K J$, o sea, $C F K J(M, V, R, D, G E)=h(g K J)$. La función $\mathrm{h}$ depende del marco legal y la legislación bancaria y comercial vigente, y puede modificarse con la creación de nuevos instrumentos y nuevas reglas en el Código de Comercio; $\mathrm{g}$ depende de la trayectoria, experiencia y tecnología de la institución financiera.

La importancia del conocimiento del agente productor y de la cadena resulta obvia para los agentes financieros externos, quienes deben diseñar contratos financieros de financiamiento ajustados a la cadena, al agente productor $y$ a la normativa vigente. La probabilidad del agente $j$ de recibir financiamiento externo del agente financiero k es igual a PKJ = (ICGj)xWKJ(Ij, Cj, Ej, $\mathrm{Xj}, \mathrm{GjP}, \mathrm{Hj}$ ). Metodológicamente, el índice WKJ debe normalizarse para que varíe entre 0 y 1 , siendo 1 el mayor valor posible que puede alcanzar el índice. Igualmente, los valores del índice ICGj deben normalizarse y sumar cien por ciento, de manera que PKJ resulte un valor promedio ponderado por la calidad de la cadena. Esa expresión recoge el efecto de las variables crediticias y no crediticias sobre el acceso al financiamiento, el control y la estructura de mercado. Resulta entonces relevante desde el punto de vista de la política económica, intervenir para mejorar las condiciones de acceso y crear nuevas condiciones (instrumentos, contratos, mecanismos de coordinación) para que los pequeños productores rurales, pequeños grupos organizados de procesadores y pequeños exportadores puedan integrarse a las cadenas globales de mercancías.

14 En esta sección se propone incorporar a la tecnología bancaria de análisis de crédito que los agentes financieros elaboren también un índice de valor crediticio de cadena como un todo. 
Para elaborar un paquete completo de servicios de financiamiento a distintos agentes productores en una misma cadena, determinar su valor crediticio y la probabilidad de cumplimiento del contrato financiero, el agente financiero externo debe conocer un amplio conjunto de información sobre los productores y las cadenas:

1. Características del agente productor y características de la cadena.

2. Tipología de los agentes productores: nuevos, medios y establecidos.

3. Tipología de las cadenas de mercancías: nuevas, medias y establecidas.

4. Gobernanza y control existente en los mercados entre segmentos conexos de la cadena como función de las cantidades y precios manejados y cualquier otra variable cuantitativa o cualitativa relevante.

Con relación al punto 1 anterior, las características del agente productor comprenden las siguientes variables: tamaño económico, edad, riqueza, educación, tamaño de la familia, información-conocimiento-experiencia (ICE) en producción, comercio, gestión y administración, innovación en producción y administración, participación de mercado, grado de control de cantidades y precios en su segmento e influencia en otros segmentos anteriores y posteriores, historial crediticio, historial comercial, propiedad de activos que sirvan como garantías y cualquier otra característica específica del producto y la cadena.

Con esa matriz de información los agentes financieros, bajo el marco legal y normativo existente, deben diseñar instrumentos o contratos financieros diferenciados para cada una de las nueve categorías, según se indica en el Cuadro 1. Cuanto más nuevo e inexperto es el agente productor y más nueva sea la cadena de mercancías, menor es la probabilidad de tener acceso al financiamiento, y si lo recibiera, las condiciones establecidas en el contrato financiero (CF) son menos favorables. Cuanto más establecido y maduro sea el agente productor y la cadena global, la probabilidad de recibir el financiamiento es mayor y las condiciones del contrato financiero (CF) son más favorables (Cuadro 1).\}

Para el agente financiero externo que analiza una solicitud de crédito de un agente productivo en una cadena, si este muestra como parte de su historial que tiene acceso al financiamiento interno, esto es, que es sujeto de crédito de otros agentes productivos de la cadena, este evento indicaría capacidad para ser sujeto de crédito formal, en cuyo caso las garantías podrían ser facturas, contratos comerciales y recibos de depósito. Para la asignación de crédito no debería importar si el agente productor está en segmentos cercanos al origen del producto (origen de la cadena, aunque dicho segmento esté compuesto por agentes que compiten en distintas regiones de un mismo país o incluso de distintos países) o si está en los segmentos cercanos al consumidor final, porque en ambos casos lo que interesa es la rentabilidad del solicitante, su disposición de pago y las garantías disponibles. 
Revista de Política Económica • EISSN: 2215-4167

Vol. 2 (1) • Julio-Enero, 2016: 1-22

DOI: http://dx.doi.org/10.15359/peds.2-1.1

Díaz Porras y Carranza

URL: http://www.revistas.una.ac.cr/politicaeconomica

Cuadro 1. Categorías de la relación productor-cadena

Probabilidad de acceso y condiciones del contrato financiero

\begin{tabular}{|c|c|c|c|c|}
\hline \multirow{2}{*}{$\begin{array}{l}\text { FINANCIAMIENTO } \\
\text { EXTERNO } \\
\text { (bancario, cooperativo, } \\
\text { otro) }\end{array}$} & & \multicolumn{3}{|c|}{ Peor $<$ Probabilidad de acceso $\left(P_{K J}\right)$ y Condiciones del $C F \rightarrow$ Mejor } \\
\hline & \multicolumn{3}{|c|}{$\begin{array}{c}\text { TIPOS DE AGENTE PRODUCTOR } \\
\text { (agricultor, procesador, exportador) }\end{array}$} & \\
\hline \multirow{4}{*}{$\begin{array}{l}\text { Peor } \\
\uparrow \\
\text { Probabilidad de acceso } \\
\left(P_{K J}\right) \\
\text { y } \\
\text { Condiciones del } C F \\
\downarrow \\
\text { Mejor }\end{array}$} & TIPOS DE CADENA & Nuevo & Medio & Establecido \\
\hline & Nueva & $\begin{array}{l}P_{K J} \text { Nula } \\
\text { Condiciones: No apli- } \\
\text { ca }\end{array}$ & $\begin{array}{l}P_{K J} \text { Baja } \\
\text { Condiciones: } \\
\text { Malas }\end{array}$ & $\begin{array}{l}P_{K J} \text { Media } \\
\text { Condiciones: } \\
\text { Medias }\end{array}$ \\
\hline & Media & $\begin{array}{l}P_{K J} \text { Baja } \\
\text { Condiciones: } \\
\text { Malas }\end{array}$ & $\begin{array}{l}P_{K J} \text { Media } \\
\text { Condiciones: } \\
\text { Medias } \\
\end{array}$ & $\begin{array}{l}P_{K J} \text { Alta } \\
\text { Condiciones: } \\
\text { Buenas }\end{array}$ \\
\hline & Establecida & $\begin{array}{l}P_{K J} \text { Media } \\
\text { Condiciones: } \\
\text { Medias }\end{array}$ & $\begin{array}{l}P_{K J} \text { Alta } \\
\text { Condiciones: } \\
\text { Buenas }\end{array}$ & $\begin{array}{l}P_{K J} \text { Muy alta } \\
\text { Condiciones: } \\
\text { Muy buenas }\end{array}$ \\
\hline
\end{tabular}

Fuente: Carranza y Díaz, 2015

\section{Conclusiones}

El financiamiento en las cadenas globales de mercancías debe resolver los viejos problemas de información asimétrica y de reducción de los porcentajes de mora de la cartera de crédito, en el marco de la normativa comercial y bancaria vigente en cada mercado o país. Los avances más recientes en el análisis de cadenas globales de mercancías han introducido un nuevo elemento en la práctica financiera de análisis de solicitudes de crédito.

Asegurar el financiamiento a un agente de la cadena, aunque sea el más grande, sin considerar las necesidades de financiamiento de otros agentes dentro del mismo segmento, o en otros segmentos, pone en riesgo el éxito total de la cadena global y, por ende, el éxito individual de los agentes en toda la cadena. Esto se ha resuelto con la acción de diversos agentes financieros atendiendo distintos agentes en distintos segmentos de la cadena, pero la práctica tradicional de la banca debe modificarse considerando muchas de las justificaciones expuestas en este artículo. Sin embargo, dichas modificaciones no corresponden exclusivamente a ese gremio, ya que muchos cambios no pueden llevarse a cabo sin reformas en el marco legal y en los derechos de propiedad de cada país.

Para que pequeños productores rurales, pequeños grupos organizados de procesadores y pequeños exportadores puedan tener acceso al financiamiento e integrarse a las cadenas globales 
de mercancías, es necesario crear instrumentos financieros y aprobar las reformas legales que se requieran para:

1. Permitir asignar recursos prestables (fondos) a un agente que no esté obligado a comprometer su activo y patrimonio por la totalidad de la suma recibida si forma parte de un mecanismo de distribución (Special Purpose Vehicle o SPV) a otros agentes, quienes reciben parte de los beneficios del financiamiento y son corresponsables de la garantía del préstamo y del reembolso del mismo a conveniencia del agente financiero.

2. Instrumentalizar la presión de grupo (peer pressure) y efectuar un seguimiento solidario del uso del crédito que permita evitar el comportamiento deshonesto (moral hazard) o daño moral y patrimonial al agente financiero.

3. Crear seguros de producción y seguros de crédito como complemento o en sustitución de garantías para enfrentar el incumplimiento de pagos.

4. Otorgar créditos individuales y créditos a clientes sindicados mediante una figura legal pertinente, tipo vehículo de propósito especial (SPV).

5. Crear las figuras legales e instrumentos financieros necesarios para otorgar créditos por agentes financieros sindicados o grupos de agentes financieros que se unen para hacer frente, mancomunando sus recursos, a alguna necesidad financiera en la actividad productiva.

La sociedad del conocimiento que caracteriza la actual fase capitalista de desarrollo genera una enorme presión social en el sentido de ampliar los desequilibrios entre los participantes del proceso económico. La creciente importancia del conocimiento como factor esencial en la producción de valor genera un rompimiento estructural de los fundamentos materiales del crecimiento económico, tradicionalmente asociado con la dotación de factores como tierra, trabajo o capital. Todo lo anterior ha provocado una desmaterialización de la producción y una clara ampliación de las brechas entre sociedades, regiones, clases sociales e individuos.

La acumulación y desacumulación de conocimiento a una velocidad sin parangón en la historia humana generan un stress sobre las bases y fundamentos del sistema productivo y, en general, del sistema social de creación, acumulación, intercambio y reproducción del conocimiento. Este se entiende como un sistema de innovación que integra socialmente los componentes relacionados con la creación y uso económico del conocimiento, a través de aplicaciones diversas a la esfera productiva.

Las posibilidades para el desarrollo sistémico de círculos virtuosos en materia de acumulación y expansión social del conocimiento condiciona la creación de sociedades de aprendizaje alto y con índices de creciente competitividad y desarrollo económico. Se trata de procesos de crecimiento sustentable de la base material del bienestar en las sociedades capitalistas de nuestro tiempo, a saber, el proceso de creación y destrucción creativa de conocimiento (Vargas, 2010,p. 8). 
Revista de Política Económica • EISSN: 2215-4167

Vol. 2 (1) • Julio-Enero, 2016: 1-22

DOI: http://dx.doi.org/10.15359/peds.2-1.1

Díaz Porras y Carranza

URL: http://www.revistas.una.ac.cr/politicaeconomica

En particular, cuando nos referimos al sector energía, la forma en que se incorporan los objetivos sociales o ambientales en la función objetivo a maximizar clásicamente hace referencia al mercado. En este particular, tradicionalmente los economistas nos hemos ocupado de estudiar la estructura del mercado -la forma en cómo se determinan los precios y cantidades dentro de un mercado-, suponiendo como un dato el contexto y las condiciones del entorno que dan lugar a esa particular forma de organización y estructura del mercado. Es claro que por el lado de la oferta, la función de producción neoclásica convencional prácticamente da por supuesto el tema tecnológico, asumiendo que es un bien común o que es exógeno al modelo de producción. De lo anterior podríamos decir que la trayectoria tecnológica sería exógena dentro de esta escuela de pensamiento.

El estudio de la estructura del mercado nos conduce desde la tradicional versión neoclásica de competencia perfecta, hasta las estructuras no competidas del mercado: los extremos del monopolio o monopsonio, así como las variaciones monopsonísticas y monopolísticas que se acostumbran describir en la literatura económica de los sectores de infraestructura (Varian, 1999).

Una vez definida la estructura de mercado y el interés más o menos estratégico del bien o servicio para el conjunto de la sociedad, la labor regulatoria define lo que se conoce como la arquitectura del mercado, es decir, las reglas del juego sobre cómo operan las actividades de producción, transporte y consumo de bienes y servicios dentro de dicho mercado. Generalmente se define como función objetivo a maximizar las ventajas en el precio, la calidad y en algunos casos la accesibilidad del bien, dejando de lado otros objetivos de mediano y largo plazo, tales como la seguridad de la oferta, la sostenibilidad ambiental o social y, por supuesto, muy pocas veces se determinan metas de diversificación de riesgos.

No se puede entender un mercado sin comprender sus dos partes, así como no se puede decir mucho o poco sobre los resultados -performance- del mercado si no se interpreta en el contexto más amplio del performance. generalmente o resultados de eficacia del sistema democrático - político- en hacer valer los intereses de la ciudadanía. Si bien es cierto la tecnocracia burocrática puede elevar los costos de transacción del sistema en proporciones muy altas, la eficiencia final en el cumplimiento de los fines y objetivos de la regulación también permite evaluar el proceso de elaboración de reglas e instituciones del país en su contenido democrático (Vargas, 2002).

De esta forma, un buen funcionamiento del mercado y de su regulación -su arquitecturase asociaría a un buen funcionamiento del sistema democrático (Hvelplund, 1999). Si ambas cosas son correctas, las preguntas tradicionales de si regular o no regular, qué bienes regular y cuándo, así como las características particulares de los procesos regulatorios de cada país, tienen su fundamento en la particular forma en que asume la selección democrática de los representantes populares ante el parlamento, como expresión de la voluntad de la ciudadanía. Al respecto, lo que parece extraño es que si los objetivos de seguridad energética y de sostenibilidad social y ambiental son de alto interés de la ciudadanía, existen claramente fallas en el entorno interinstitucional que no permiten generar regulaciones y condiciones para el proceso de regulación cotidiana que garanticen resultados efectivos respecto a dichos objetivos en la operación de los sistemas eléctricos en la cotidianeidad centroamericana (Vargas, 2010). 
Otro aspecto a destacar son las características que asume el proceso de diseño de un modelo regulatorio, es decir, la forma particular de definir la arquitectura de la regulación del mercado. En este campo reviste mucha importancia ver el proceso de aprendizaje institucional dentro del mercado, así como los procesos de aprendizaje trasladados desde otras regiones del mundo. Esta segunda parte se refiere más al cómo y dónde regular, quién regula y cuándo se regula o no. Sobre este particular, reviste de importancia destacar que las funciones objetivo de la regulación existente se fundamentan en la optimización del corto plazo, generando presión por el referente precio y en algunos casos la calidad o accesibilidad del servicio.

Estas funciones objetivo muchas veces se contraponen a objetivos de diversidad tecnológica, que podrían permitir el acceso de nuevas fuentes energéticas con curvas de aprendizaje menores y, como tal, costos un tanto superiores a corto plazo, lo mismo que objetivos como el medio ambiente o cambio climático, que obligarían a medidas de restricción directa del uso de ciertas opciones tecnológicas en concordancia con un uso sostenible de los recursos. De igual forma, los procesos de eficiencia y de sustitución de energía podrían requerir de premios y castigos mayores que garanticen efectividad de las políticas y el traslado en el tiempo de las ventajas de usar un menor coeficiente de intensidad energética por parte de los países (Sterling, 1999).

En muchas ocasiones las inversiones corporativas ya realizadas tanto por empresas públicas como por empresas privadas, se contraponen con la deseabilidad de cambio en el sector, debido a amortizar costos hundidos de inversiones del pasado que deberían dejarse atrás y fortalecer un proceso de cambio tecnológico e institucional más favorable a las trayectorias limpias en materia energética. El poder corporativo privado y público de dichas empresas o de grupos sindicales o de presión muchas veces se convierte en una presión fuerte ante el cambio, evitando o reduciendo los márgenes del mismo (Kemp, 1995).

En este contexto, el cambio tecnológico es un proceso gradual y coevolutivo con alta dependencia del pasado. Las transformaciones institucionales afectan claramente la trayectoria de cambio en el sector de igual forma que se generan importantes efectos en el patrón de especialización de los países (Lundvall, 1999). Es de esperar un proceso de aprendizaje gradual e interactivo muy influenciado por la trayectoria del pasado. En la siguiente sección intentaremos destacar la evolución reciente de dicha trayectoria para la región centroamericana en los últimos años.

\section{Bibliografía referenciada}

Carranza, C. (2001). Crédito Agropecuario y Sostenibilidad de la Agricultura. Revista Economía y Sociedad, 16, 25-44. Recuperado de: www.revistas.una.ac.cr/index.php/economia/article/

Carranza, C. (2002). Aplicación del modelo Tobit a la estimación de la oferta de crédito agropecuario en Costa Rica. Revista Economía y Sociedad,19, 109-126. Recuperado de: http://www.revistas.una.ac.cr/index.php/economia/article/view/1189 
Carranza, C. y Díaz, R. (2005). Mercados financieros en las cadenas agroindustriales de mercancías: aproximación conceptual. Revista ABRA,34, 53-83. Recuperado de: http:// www.revistas.una.ac.cr/index.php/abra/article/view/4194

Chalmers, G., Gálvez, E., Tiffen, P. y Wenner, M. (2007). Lecciones aprendidas en el financiamiento de las cadenas agrícolas de valor. En Quirós, R. Financiamiento de las cadenas agrícolas de valor. San José, Costa Rica: Academia de Centroamérica. Recuperado de: http://unpan1. un.org/intradoc/groups/public/documents/ICAP/UNPAN028340.pdf

Fries, R. y Akin, B. (2004). Value Chains and Their Significance for Addressing the Rural Finance Challenge. 1st ed. [ebook] Washington, DC: Accelerated Microenterprise Advancement Project. Retrieved from: http://pdf.usaid.gov/pdf docs/Pnadi913.pdf

Gereffi, G. y Korseniewicz, M. (1994). Commodity chains and global capitalism. USA: Greenwood Publishing Group.

Hess, C., Springer-Heinze, A., Bertenbreiter, W., Jarchau, P. y Braun, P. (2007). Services for Rural Development. Agriculture, Fisheries and Food, Division 45. Bonn, Germany.

Hvelplund, F. (1999). Energy planning, democracy and innovation: the Danish and East German example. Paper presentado en la Conferencia Internacional SUDESCA, Costa Rica, February.

Jansen, A. (2007). Value Chain Finance. Understanding \& Increasing Access. A concept paper. Washington, DC: Accelerated Microenterprise Advancement Project. Retrieved from: https://www.microlinks.org/sites/microlinks/files/resource/files/ML5901 value chain finance concept paper dec 2007.pdf

Johnston, C. (2007). Value Chain Governance and Access to Finance: Maize, Sugar Cane and Sunflower Oil in Uganda Washington, DC: Accelerated Microenterprise Advancement Project. Retrieved from: http://www.ruralfinanceandinvestment.org/sites/default/ files/1205146927823 Final microREPORT 88 Value Chain Governa-619578901.pdf

Kemp, R. (1995). Environmental policy and technical change: A comparison of the technological impact of policy instruments. [Política medioambiental y el cambio técnico: Una comparación del impacto tecnológico de los instrumentos de política]. (Tesis de doctorado). Maastricht University. Maastricht.

Lundvall. B.A. (1999). Nation states, social capital and economic development $-\mathrm{a}$ system's approach to knowledge creation and learning [Estado nación, capital social y desarrollo económico- un enfoque sistémico de la creación del conocimiento y el aprendizaje]. Conferencia SUDESCA, San José, Costa Rica. 
Meyer, R. L. (2007). Analyzing and Financing Value Chains: Cutting Edge Developments in Value Chain. Presentation at the 3rd African Microfinance Conference: New Options for Rural and Urban Africa, (August), 20-23. Retrieved from: http://www.ruralfinanceandinvestment. org/sites/default/files/1193502947567 Meyer analysing value chains.pdf

Royal Tropical Institute (KIT), International Institute of Rural Reconstruction (IIRR) y FOROLACFR. (2010). Financiamiento de cadenas de valor: Más allá de las microfinanzas para emprendedores rurales. Lima: International Institute of Rural Reconstruction. Recuperado de: http://www.ruralfinanceandinvestment.org/sites/default/files/libroFinanciamiento de Cadenas de Valor.pdf

Shwedel, K. (2007). Financiamiento de las cadenas de valor: una estrategia para lograr racionalidad, competitividad e integración. San José, Costa Rica: Academia de Centroamérica. Recuperado de: https://www.academiaca.or.cr/wp-content/uploads/2017/06/doc 4768.pdf

Sterling, A., \& Mayer, S. (1999). Rethinking Risk: a pilot multicriteria mapping of a genetically modified crop in agricultural systems in the UK. Reino Unido: Universidad de Sussex. Recuperado de: http://users.sussex.ac.uk/ prfh0/Rethinking\%20Risk.pdf

Sucre-Reyes, M. A. (2014). Finance, growth and social fairness: Evidence for Latin America and Bolivia Tilburg: CentER, Center for Economic Research. Retrieved from: https://pure.uvt. nl/ws/files/3602078/FinalThesis M.Sucre all in greys Corrected 27May.pdf

UNCTAD. (2004). Financing Commodity-Based Trade and Development: Innovative Agriculture Financing Mechanisms. Reporte preparado para la secretaria de la UNCTAD. Retrieved from: http://www.jstor.org/stable/10.2307/41392810

Vargas, L. (2002). Competitiveness, innovation and democracy: Space for clean energy within electricity reforms (Tesis de doctorado). Universidad de Aalborg: Dinamarca.

Vargas, L. (2010). Proyecto regulación, innovación y ambiente (REINA). Centro Internacional de Política Económica para el Desarrollo Sostenible: Heredia

Varian.H (1999). Microeconomía Intermedia (5ta Edición). Barcelona: Antoni Bosch.

Zeller, M. (2005). A fresh look at Rural \& Agricultural Finance. Issue I. United States Agency for International Development. Office of Agriculture and Microenterprise. Retrieved from: https://afraca.org/?wpfb dl=220 